\title{
Nestlé Lebensmittelanalytik in einem globalen Netzwerk
}

\author{
Ralf Kurt Günter Kuchenbecker
}

Nestlé Deutschland AG, Frankfurt / Polling, Nestlé Quality Assurance Center Weiding

\section{Zusammenfassung}

Nestlé -ein forschendes Unternehmen in der Familie der großen Lebensmittelhersteller- nutzt in Ihren Forschungs- Entwicklungs- Produktions- und Analytikzentren Sensoren um Lebensmittel für Mensch und Tier herstellen zu können. Ohne Sensoren kann die Erzeugung von Lebensmitteln nicht erfolgen. Dieser Vortrag hat nicht zum Ziel, Sensoren in Ihrem Aufbau detailliert zu erklären, soll aber einen allgemeinen Überblick verschaffen, wie vielfältig die oft nicht mehr bemerkte Überwachung durch Sensoren im Wertschöpfungsprozess eines Lebensmittels geworden ist. Rohstoffe werden weltweit angebaut und lokal zu Fertigprodukten transformiert (Frankreich benutzt eine treffende Beschreibung: Societé Industrielle Transformation Produits Agricole SITPA), um danach regional und global in den Vertrieb zu gehen. Dieser über Sensoren unterstützte Wertschöpfungsprozess ist dem Prinzip unterstellt, sichere Lebensmittel zu erzeugen. Über Konsumentenzufriedenheit und wettbewerbsfähige Qualität ist Markenakzeptanz geschaffen, die die Basis für ein erfolgreiches Markenunternehmen darstellt.

Ein Schlüsselprozess in dieser Wertschöpfungskette stellt die Analytik dar, die als Unterstützungsprozess mit dem Ziel, sichere und gesunde Lebensmittel herzustellen, zu verstehen ist. Nestlé verfolgt dabei das Konzept durch gezielte Rohstoffauswahl und Überwachung bei der Produktion zeitraubende und aufwendige Endproduktuntersuchungen zu vermeiden.

\section{Begriffserklärung Sensor und der Bezug zur Analytik}

\subsection{Wie grenzt sich der Begriff Sensor von den Begriffen Detektor, Messaufnehmer und Messgerät oder Prüfmittel ab ?}

\subsubsection{Dudendefinitionen}

sensus $=$ Gefühl, Empfindung,Wahrnehmung

Sensor : Hochempfindliches Nachweis- und Kontrollgerät (z.B. für Licht und Temperaturschwankungen)

\subsubsection{Definition aus meinem Studium der Chemie und Biologie}

Ein Sensor ist einer der ersten Bausteine in einer Wahrnehmungs- bzw. Messkette, der dazu beiträgt, dass eine Eingangsgröße bzw. ein Oberflächenvorgang in ein neuronales, analoges oder digitales Signal umgewandelt werden kann.

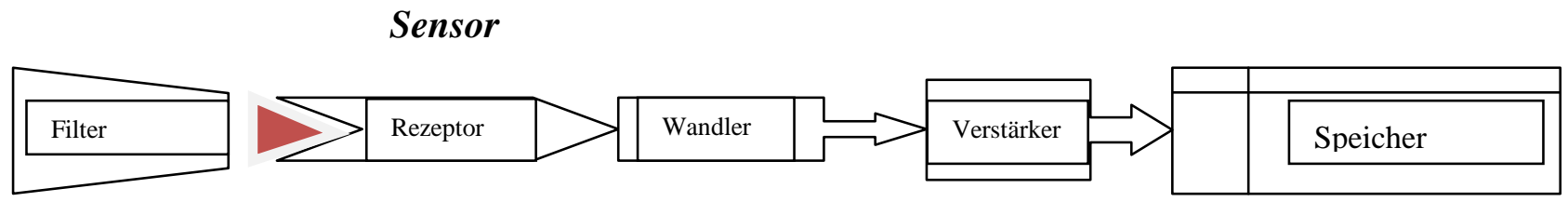

Bild 1 schematische Darstellung einer Messkette mit Speicherelement 


\subsection{Analytik}

Analytik bedeutet laut Dudendefinition „Zerlegungslehre“. Sie verfolgt das Ziel, mittels systematischer Verfahren, z. B. Lebensmittelkomponenten und nicht erwünschte Rückstände über Sensoren, die als Komponenten von Messumformern zu verstehen sind, qualitativ und quantitativ zu bestimmen.

\section{Sensoren in der Wertschöpfungskette eines Lebensmittels (Von G-Protein zu G-Protein)}

Der Lebenszyklus eines Lebensmittels vom Acker bis zur Gabel startet bereits vor dem Anbau der Rohstoffe. Zur Erzeugung hochwertiger Lebensmittel z.B. für besonders schützenswerte Konsumentengruppen (Kleinkinder) ist es notwendig, geeignete Anbauflächen und Rohstofferzeuger auszuwählen. Diese Flächen sind aufgrund zunehmender Konsumentenerwartungen weltweit auch nicht mehr unbegrenzt verfügbar. Das zunehmende Gesundheitsbewusstsein der Konsumenten, insbesondere in den hochtechnisierten Gesellschaften, der aber gleichzeitig seit vielen Jahren zunehmende Trend der Megapolisierung der Weltbevölkerung und die damit notwendige Lebensmittellogistik, fordert uns auf, die Lenkung von Risiken in den Phasen der Lebensmittelerzeugung, Lebensmittelverarbeitung, Lebensmittelvertrieb (inklusive Logistik) einschließlich Lebensmittelkonsum, verstärkt unter dem Aspekt der Analytik im Auge zu behalten.

Sind Dioxine im Schweinefleisch, EHEC Bakterien auf Obst und Gemüse bzw. Sprossen, Listerien auf Melonen Botschafter eines drohenden Kollapses der Lebensmittelkontrolle bzw. Analytik. Dieser Vortrag wird keine Lösung präsentieren, gibt aber den Entwicklern von Sensoren einen Überblick, was zur Zeit getan wird, Konsumenten durch analytische Vorgehensweisen vor Gefährdungen und Risiken zu bewahren. Sensoren leisten hier einen entscheidenden Beitrag dieses Ziel zu erreichen.

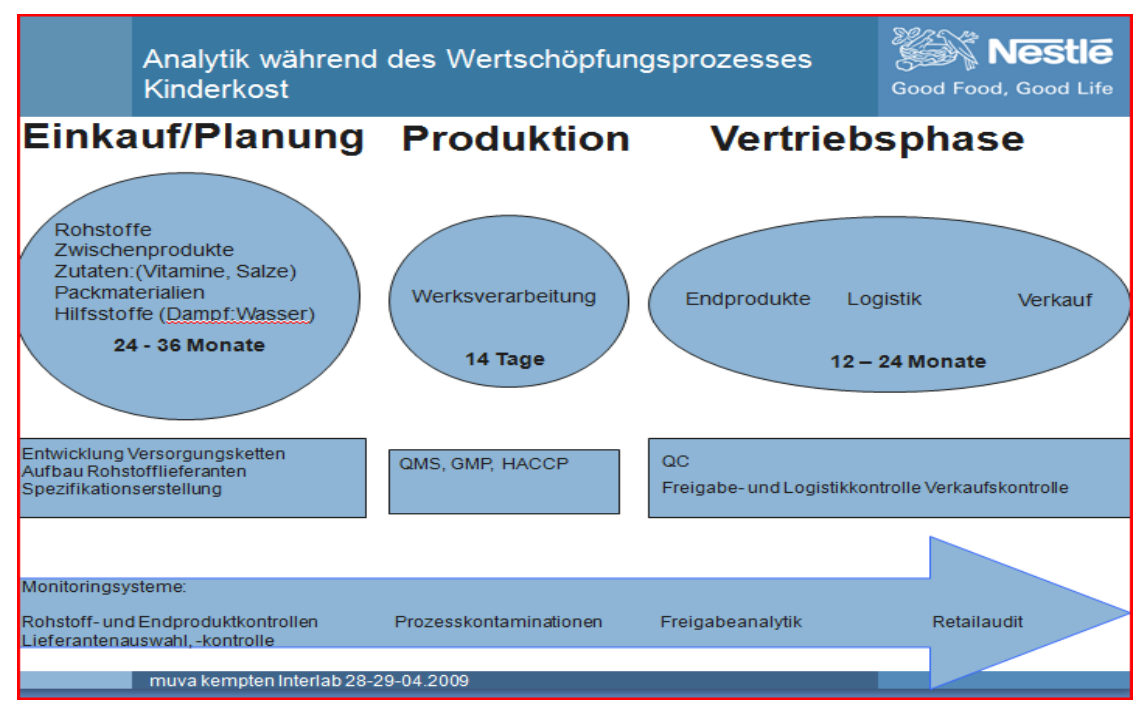

Bild 2 Der Lebenszyklus eines Lebensmittels und seine Begleitprozesse zur Sicherstellung der Qualität und Sicherheit Kinderkost (Quelle:Vortrag Interlabtagung 2009 MUVA Kempten Dr. Ralf Kuchenbecker) 


\subsection{Rohstoffe Anbau bis zur Produktionsfreigabe}

\subsubsection{Rohstoffanbau}

Sensoren leisten wichtige Beiträge zur Einschätzung des Schädlingsdruck in Obstplantagen, weiterhin wird durch die eingeleitete Signalkette und das dadurch verbundene Fehlverhalten der Schadinsekten, die Fortpflanzung behindert. Über die Einschätzung des Schädlingsdrucks kann über den moderaten Einsatz von Insektiziden oder Verzicht der Insektizidanwendung entschieden werden, den Ernteertrag sicherzustellen.
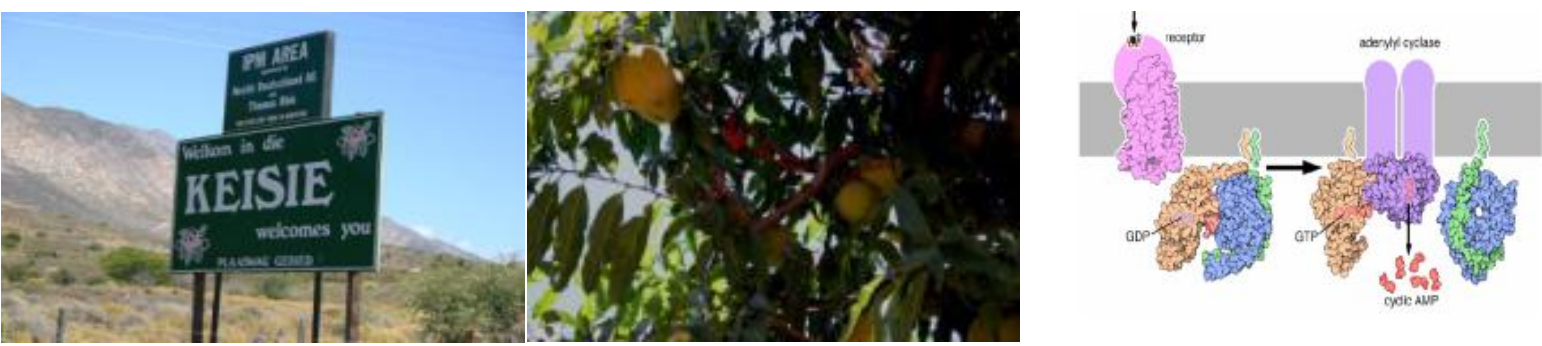

Bildgruppe 3: Pheromon basierende Verwirrungsmethode zur Eindämmung von Schadinsekten (Eigentum Dr. Ralf Kuchenbecker) mit einer Beschreibung der molekularen Signalkaskadierung, der Transmembranreceptor stellt den eigentlichen Sensor dar; Darstellung aus dem Biochemielehrbuch Stryer 6. Auflage G Protein aktivierte Signalkette zur Ausschüttung von C AMP.

\subsection{Rohstoff- und Endproduktanalytik}

Neben der Analytik zur Bestimmung der Grundzusammensetzung von Lebensmittelrohstoffen werden innerhalb der Nestlé in einem weltweit verbundenen Labornetzwerk von 27 Laboratorien, Rohstoffe und Fertigprodukte auf wertgebende (Nutritional Facts) und wertzerstörende Bestandteile (Pestizide, Umweltund Prozess- Kontaminanten, Toxine, Migrationsmoleküle aus Packmaterialien, GMO Verunreinigungen und mikrobiologische Parameter untersucht. In Abhängigkeit von der Risikobewertung der Rohstoffe werden bis zu 1700 Prüfparameter zur Freigabe eines Rohstoffes ermittelt und bewertet. Diese Daten werden in einer zentralen Datenbank verwaltet, um Anbau- und Einkaufstrategien zu entwickeln, Risiken hinsichtlich Qualität und rechtlicher Vorgaben in den Märkten zu minimieren bzw. ausschließen zu können.

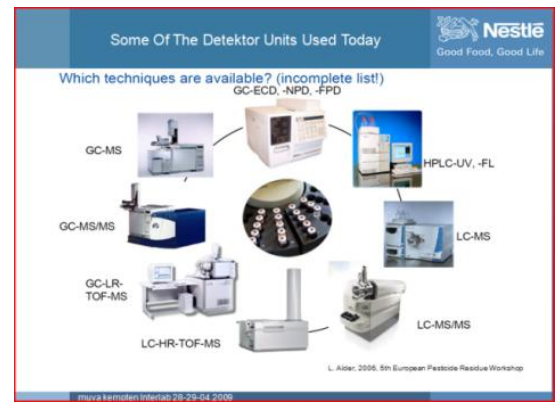

Bild 4: Darstellung eines NQAC Analytik Inventars zur Rückstandsuntersuchung von Lebensmittelrohstoffen und Endprodukten

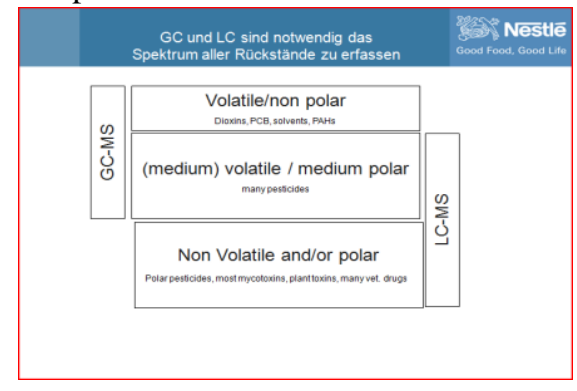

Bild 5 Potentialabschätzung jetziger und zukünftiger GC Anwendungen, LC Anwendungen werden ein stärkeres Gewicht bekommen 


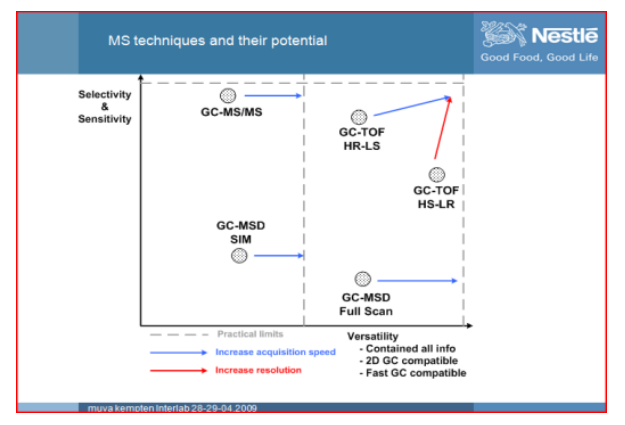

Bild 6 GC Detektor Kopplungen (Quelle: Nestlé NRC Lausanne 2009)

Die folgende Tabelle solleinen groben Überblick der zur Zeit verwendeten Sensoren / Detektoren im Bereich Lebensmittelanalytik darstellen.

Tafel 1 Sensoren in der Lebensmittelsanalytik

\begin{tabular}{|c|c|}
\hline Analysengruppe & Sensoren bzw. Meßprinzip \\
\hline Packmitteluntersuchungen & GC und LC Techniken \\
\hline Nutritional Facts & $\begin{array}{l}\text { Nasschemische Verfahren ( Titrationsautomaten } \\
\text { gekoppelte Elektrodensysteme Potential bzw. } \\
\text { Impedanztechniken }\end{array}$ \\
\hline Nutritielle Elemente & $\begin{array}{l}\text { optische Detektoren Photomultiplier bzw. CCR } \\
\text { Detektoren, siehe ICP OES Detektoren, FET`S } \\
\text { Potentialsensoren (Ionenselektive Elektroden) }\end{array}$ \\
\hline Schwermetalle & Photomultiplier bzw, CCR Detektoren \\
\hline Pesticide & $\begin{array}{l}\text { ECD, NPD, MS Kopplungen mit diversen Quatropol / } \\
\text { Octopol vorgeschalteten Filtereinheiten, heutzutage } \\
\text { immer noch auf offenen Photomultipliern basierend }\end{array}$ \\
\hline Allerenge & $\begin{array}{l}\text { optische Sensoren (Photodioden, Photozellen) Surface } \\
\text { Plasmon Techniken (massenselektiver Detektor) }\end{array}$ \\
\hline Antibiotika & $\begin{array}{l}\text { MS Techniken, ELISA gekoppelte optische Detektion } \\
\text { Surface Plasmon }\end{array}$ \\
\hline
\end{tabular}

\section{Rohstoffverarbeitung bis zur Endproduktfreigabe}

Der Produktionsprozess wird nach der Validierung in normierter Form kontrolliert durchgeführt . Neben klassischen Überwachungen zu Druck, Temperatur, pH Wert, Leitwert, Strömungsgeschwindigkeit gewinnen bildgebende Sensorsysteme an zunehmender Bedeutung bei der Rohstoffsortierung, Packmittelinsspektion, Fremdkörperkontrolle und Dichtigkeitsüberwachung Vakuum verpackter Lebensmittel. Mittels CCR Photochip Sensortechnik sind mit hoher Taktrate $100 \%$ Kontrollen, die nicht zerstörerisch sind durchzuführen. 
Tafel 2 Sensoren in der Lebensmittelproduktion

\begin{tabular}{l|l}
\hline Prozeßschritte & Sensoren bzw Kontrollprinzip \\
\hline Packmittelinspektionen & $\begin{array}{l}\text { Otpische Geräte (CCR Chip Kamera) bzw, } \\
\text { Impedanztechniken, menschliches Auge }\end{array}$ \\
Rohstofftransporte über Leitungen und Rohre & $\begin{array}{l}\text { IDM Magnet, Optische Kontrolle, Schallsensoren } \\
\text { (Fremdkoerperdetektion und Ausschleusung) }\end{array}$ \\
$\begin{array}{l}\text { Abfüllungen Behälter Container, Primäre Packmittel } \\
\text { Vichtigkeiten von Fertigverpackungen }\end{array}$ & $\begin{array}{l}\text { Druck Optische Kontrolle Impedanz } \\
\text { optische Systeme, } \\
\text { Schallsensoren ( Frequenzanalysatoren) }\end{array}$ \\
Haltbarmachung & $\begin{array}{l}\text { Temperatur, pH-Wert } \\
\text { Etikettierung }\end{array}$ \\
CIP Vorgänge & $\begin{array}{l}\text { Videooptische Kontrolle mit Highspeed Kameras } \\
\text { Trübungssensoren, Druck, Temperatur; } \\
\text { Leitwertsensoren }\end{array}$ \\
\hline
\end{tabular}

\section{Logistik, Vertrieb, Konsum}

Bei kühlbedürftigen Produkten sind heute Datenlogger (Funktransponder) zur Temperaturregistrierung die Regel, um Unterbrechungen der Kühlkette nachweisen zu können. ECR kann wertvolle Informationen zur Optimierung der zeitnahen Produktion von Lebensmitteln liefern.

\subsection{Retailphase}

Temperatur und Marktprobenüberwachung

\subsection{Die Geschmackswahrnehmung}

Lebensmittel werden akzeptiert, wenn (S) ie schmecken; dies basiert auf G - Proteinen als Sensoren.

[1] Bild 2 Quelle: Vortrag Interlabtagung 2009 MUVA Kempten Dr. Ralf Kuchenbecker

[2] Bildgruppe 3 Quelle: Bilder Nestlé Keisie Anbauregion für Früchte Südafrika ; G-Protein Darstellung aus Biochemielehrbuch L. Stryer 6. Auflage

[3] Bilder 4-6 Quelle: Nestlé NRC und NQAC Weiding Vortragskonzepte 